\title{
INDEX OF VOLUME XXXIII
}

Adams, E. P. See Reviews, under Birtwistle, Bligh, Haas, Peirce.

Allen, E. S. See Reviews, under Stenström.

Ayres,W. L. A New Characterization of Plane Continuous Curves, 201.

- Concerning the Boundaries of Domains of a Continuous Curve, 565.

Bateman, H. A Cubic Curve connected with Two Triangles, 45.

- See Reviews, under Eyraud, Gonseth, Walmsley.

Bell, E. T. A Diophantine Automorphism, 71.

Cauchy's Cyclotomic Function and Functional Powers, 416.

Bernstein, B. A. The Dual of a Logical Expression, 309.

- Reports of Meetings of the San Francisco Section: October Meeting, 2; April Meeting, 269; June Meeting, 513.

- See Reviews, under Zaremba.

Birkhoff, G. D. A Mathematical Critique of some Physical Theories, 165.

Brown, B. H. See Reviews, under Kommerell.

Cajori, F. See Reviews, under Huygens, Neugebauer.

Camp, C. C. A Method for Accelerating the Convergence in the Process of Iteration, 209.

Campbell, A. D. Three-Parameter and Four-Parameter Linear Families of Conics in the Galois Fields of Order $2^{n}, 608$.

Carmichael, R. D. See Reviews, under Chwolson, Einstein, Heyl, Hobson, Sommerfeld.

Carson, J. R. A Generalized Two-Dimensional Potential Problem, 296.

Chitrenden, E. W. On the Metrization Problem and Related Prcblems in the Theory of Abstract Sets, 13.

Cole, F. N. See Fiske, T. S.

Conwell, G. M. See Reviews, under Bouligand, Cailler, Müller.

Coolidge, J. L. Corrado Segre, 352.

Copeland, A. H. Note on the Fourier Development of Continuous Functions, 689.

Curtiss, D. R. See Reviews, under Osgood.

Davis, H. T., and Kirkham, W. J. A New Table of the Zeros of the Bessel Functions $J_{0}(x)$ and $J_{1}(x)$ with Corresponding Values of $J_{1}(x)$ and $J_{0}(x), 760$.

Dickson, L. E. Integers Represented by Positive Ternary Quadratic Forms, 63.

- A Generalization of Waring's Theorem on Nine Cubes, 299.

_ Extensions of Waring's Theorem on Fourth Powers, 319.

- All Positive Integers are Sums of Values of a Quadratic Function of $x, 713$.

Dines, L. L. Linear Inequalities in General Analysis, 695.

Dodn, E. L. The Probability Law for the Intensity of a Trial Period, with Data subject to the Gaussian Law, 681. 
Dresden, A. Reports of Meetings of the American Mathematical Society: December Meeting in Chicago, 153; April Meeting in Chicago, 385.

Емсн, A. On the Mapping of the Sextuples of the Symmetric Substitution Group $G_{6}$ in a Plane upon a Quadric, 745 .

See Reviews, under Willers.

Ettlinger, H. J. On Continuity in Several Variables, 37.

—- See Reviews, under Riemann, Schlesinger.

Evans, G. C. See Reviews under Lévy.

Fiske, T. S. Frank Nelson Cole, 773.

Fite, W. B. Bequests to the Society, 522.

FORT, T. An Elementary Proof by Mathematical Induction of the Equivalence of the Cesàro and Hölder Sum Formulas, 301.

Foster, M. C. Congruences of Lines of Special Orientation relative to a Surface of Reference, 750.

Franklin, P. A Theorem of Frobenius on Quadratic Forms, 447.

- Analytic Functions with Assigned Values, 461.

Garver, R. The Binomial Quartic as a Normal Form, 677.

Gehman, H. M. See Reviews, under Hausdorff.

Glover, J. W. See Reviews, under Boehm.

Gourin, E. See Ritt, J. F.

Gronwall, T. H. See Reviews, under Charbonnier.

Hewes, L. I. See Reviews, under d'Ocagne.

Hildebrandt, T. H. See Reviews, under Rothe.

- The Second Madison Colloquium, 663.

Hollcroft, T. R. Singularities of the Hessian, 90.

- See Reviews, under Pasch.

Hotelling, H. An Application of Analysis Situs to Statistics, 467.

InGOLD, L. Report of the Nineteenth Regular Meeting of the Southwestern Section, 162.

See Reviews, under Falckenberg.

Kirkham, W. J. See Davis, H. T.

Knaster, B., and Kuratowski, C. A Connected and Connected im Kleinen Point Set which Contains No Perfect Subset, 106.

Koopman, B. O. On Analytic Solutions of Differential Equations in the Neighborhood of Non-Analytic Singular Points, 341.

Kuratowski, C. See Knaster, B.

Kuratowski, C., and Zarankiewicz, C. A Theorem on Connected Point Sets, 571.

Lane, E. P. The Asymptotic Osculating Quadrics of a Curve on a Surface, 195.

See Reviews, under Fubini.

LANGer, R. E. Three Theorems on Closure of Biorthogonal Systems of Functions, 97 .

LANGFORD, C. H. On Inductive Relations, 599.

- An Analysis of some General Propositions, 666.

Lenmer, D. H. Tests for Primality by the Converse of Fermat's Theorem, 327.

Lehmer, D. N. A Theorem on Factorization, 35. 
Longley, W. R. See Reviews, under Fabry, Haag, Hahn, Zoretti.

Mandelbrojt, S. A Class of Transcendental Numbers, 413.

March, H. W. The Heaviside Operational Calculus, 311.

Merriman, G. M. On certain Sufficient Conditions for the Convergence and Cesàro Summability of the Allied Series of a Double Fourier Series, 576.

Miller, G. A. Determination of the Number of Subgroups of an Abelian Group, 192.

- Substitutions which Transform a Regular Group into its Conjoint, 701.

Mitchell, H. H. See Reviews, under Chatelet.

Mitchell, U. G. See Reviews, under Whetham.

Moore, C. N. See Reviews, under Bernstein, Buhl.

Moore, L. T. See Reviews, under Hasse.

Murnaghan, F. D. The Cauchy-Heaviside Expansion Formula and the Boltzmann-Hopkinson Principle of Superposition, 81.

- See Reviews, under Bouligand, von Ignatowsky, Kafka, Spielrein.

Murray, F. H. Invariant Relations, 189.

PAGE, L. See Reviews, under Ferrier.

Pierpont, J. On a Generalization of the Secular Equation, 294.

Raudenbush, H. W. On Hilbert's Thirteenth Paris Problem, 433.

Raynor, G. E. Generalization of the Beltrami Equations to Curved $n$-Space, 435.

Reynolds, C. N. See Reviews, under von Laue.

Richardson, R. G. D. Participation in the Publication of the American Journal of Mathematics, 1.

- Reports of Meetings of the American Mathematical Society: October Meeting in New York, 5; Thirty-Third Annual Meeting, 129; February Meeting in New York, 257; May Meeting in New York, 400; ThirtyThird Summer Meeting, 641.

Rietz, H. L. See Reviews, under Fisher.

RitT, J. F. On the Integration in Finite Terms of Linear Differential Equations of the Second Order, 51.

Ritt, J. F., and Gourin, E. An Assemblage-Theoretic Proof of the Existence of Transcendentally Transcendental Functions, 182.

Roever, W. H. Clarence Abiathar Waldo, 613.

Rosebrugh, T. R. A General Theorem on Quantic Determinants, 583.

Rowe, J. E. See Reviews, under Cranz.

Segre, C. See Coolidge, J. L.

Shaw, J. B. See Reviews, under Cullis.

Shoнат, J. A. A Simple Method for Normalizing Tchebycheff Polynomials and Evaluating the Elements of the Allied Continued Fractions, 427.

Shook, C. A. See Reviews, under Sternberg.

Smail, L. L. See Reviews, under Valiron.

Smith, D. E. See Reviews, under Dalton, Heath, Reymond.

Snyder, V. On a Problem in Clo'sure, 39.

Stone, M. H. The Summability of Fourier Series, 721.

Stouffer, E. B. See Reviews, under Campbell. 
Struik, D. J. On the Geometry of Linear Displacement, 523.

Thomas, T. Y. See Reviews, under Appell.

Tracey, J. I. See Reviews, under Schoenflies.

Trjitzinsky, W. J. Zeros of a Function and of its Derivative, 693.

Van Vleck, E. B. See Reviews, under Osgood.

VAN Vleck, J. H. See Reviews, under Juvet.

Wahlin, G. E. A Quadratic Algebra and its Application to a Problem in Diophantine Analysis, 221.

Waldo, C. A. See Roever, W. H.

Walsh, J. L. On the Degree of Approximation to a Harmonic Function, 591.

Ward, L. E. Functions Expansible in Series, 232.

WARD, M. A Generalization of Recurrents, 477.

Weatherburn, C. E. On Small Deformations of Curves, 58.

Weaver, J. H. Invariants of a Poristic System of Triangles, 235.

Weisner, L. A Theorem concerning Direct Products, 44.

- The Functional Equation Defining Diophantine Automorphisms, 707.

Whyburn, G. T. The Most General Closed Point Set over which Continuous Function may be Defined by certain Properties, 185.

Some Properties of Continuous Curves, 305.

- Concerning Connected and Regular Point Sets, 685.

Whyburn, W. M. On the Polynomial Convergents of Power Series, 673.

Wilder, R. L. A Point Set which has No True Quasi-Components, and which Becomes Connected upon the Addition of a Single Point, 423.

- The Non-Existence of a certain Type of Regular Point Set, 439.

Williams, H. B. Mathematics and the Biological Sciences, 273.

Wilson, E. B. See Reviews, under Eddington.

Wilson, W. A. On the Separation of the Plane by Irreducible Continua, 733.

Young, J. W. On the Partitions of a Group and the Resulting Classification, 453.

Zarankiewicz, C. See Kuratowski, C.

\section{REVIEWS}

Appell, P. Traité de Mécanique Rationnelle, volume V, T. Y. Thомas, 493.

Bernstein, S. Leçons sur les Propriétés Extrémales et la Meilleure Approximation des Fonctions Analytiques d'une Variable Réelle, C. N. MOORE, 369.

Birtwistle, G. The Quantum Theory of the Atom, E. P. Adams, 368.

Bligh, N. M. The Quantum Theory, E. P. Adams, 793.

Boehm, F. Versicherungsmathematik, I, J. W. Glover, 119.

Bouligand, G. Précis de Mécanique Rationnelle, volume I, G. M. ConWELL, 249.

Bouligand, G., et Rabaté, G. Initiation aux Méthodes Vectorielles et aux Applications Géométriques de l'Analyse, F. D. Murnaghan, 787.

Brose, H. L. See Sommerfeld, A. 
Brunschvicg, L. See Reymond, A.

Buhl, A. Séries Analytiques. Sommabilité, C. N. Moore, 120.

Cailler, C. Introduction Géométrique à la Mécanique Rationnelle, G. M. CONWELl, 249.

Campbell, J. E. A Course of Differential Geometry, E. B. Stouffer, 625.

Čech, E. See Fubini, G.

Charbonnier, P. Traité de Balistique Extérieure, volume I, T. H. GronWALL, 120.

Chatelet, A. Les Groupes Abéliens Finis et les Modules de Points Entiers, H. H. Mitchell, 790.

Chwolson, O. D. Traité de Physique, tome supplémentaire: La Physique de 1914 à 1926, première partie, traduit par A. Corvisy, R. D. CarMICHAEL, 794.

Corvisy, A. See Chwolson, O. D.

Cowper, A. D. See Einstein, A.

Cranz, C. Lehrbuch der Ballistik, J. E. Rowe, 121.

Cullis, C.E. Matrices and Determinoids, volume 3, part 1, J. B. Shaw, 618.

Dalton, O. M. The Byzantine Astrolabe at Brescia, D. E. Sмітн, 790.

Dehn, M. See Pasch, M.

Eddington, A. S. The Internal Constitution of the Stars, E. B. Wilson, 615.

Einstein, A. Investigations on the Theory of the Brownian Movement, translated by A. D. Cowper, R. C. Carmichael, 794.

Euclid. See Heath.

Eyraud, H. Les Equations de la Dynamique de l'Ether, H. Bateman, 496.

Fabry, C. Nouveau Traité de Mathématiques Générales (quatrième édition), volume 2, W. R. Longley, 781.

Falckenberg, H. Elementare Reihenlehre, L. INGOLD, 497.

Ferrier, R. Les Nouveaux Axiomes de l'Electronique, L. PAGE, 793.

Fisher, I. Mathematical Investigations in the Theory of Value and Prices, H. L. Rietz, 789.

Frank, P. See Riemann, B.

Fubini, G., e Čech, E. Geometria Proiettiva Differenziale, volume I, E. P. LANE, 113.

Gheury de Bray, R. See Reymond, A.

Gonseth, F. Les Fondements des Mathématiques, H. Bateman, 626.

Haag, J. Cours Complet de Mathématiques Spéciales, volumes I, II, III, W. R. Longley, 781.

Haas, A. Die Welt der Atome, E. P. Adams, 789.

Hahn, H., und Tietze, H. Einf ührung in die Elemente der höheren Mathematik, W. R. Longley, 781.

Hasse, H. Höhere Algebra, Band I, L. T. Moore, 251.

Hausdorff, F. Mengenlehre (zweite Auflage), H. M. Gehman, 778.

Heath, T. L. The Thirteen Books of Euclid's Elements (second edition), D. E. Smith, 246.

Heyl, P. R. The Fundamental Concepts of Physics in the Light of Modern Discovery, R. D. Carmichael, 497. 
Hobson, E. W. The Theory of Functions of a Real Variable and the Theory of Fourier's Series (second edition), volume II, R. D. CARMICHAEL, 115.

Huygens, C. Euvres Complètes, tome 15, F. CAJORI, 627.

von Ignatowsky, W. Die Vektoranalysis und ihre Anwendung in der Theoretischen Physik (dritte Auflage), F. D. Murnaghan, 787.

Juvet, G. Mécanique Analytique et Théorie des Quanta, J. H. VAN Vleck, 367.

Kafka, H. Die Ebene Vektorrechnung und ihre Anwendungen in der Wechselstromtechnik, Teil 1, F. D. Murnaghan, 787.

Kommerell, K. Aufgaben zur Synthetischen Geometrie, B. H. Brown, 498. von Laue, M. La Théorie de la Relativité, traduit par G. Letang, volume II, C. N. REYNoLDS, 498.

Letang, G. See von Laue, M.

Lévy, P. Analyse Fonctionnelle, G. C. Evans, 372.

von Mises, R. See Riemann, B.

Müller, W. Dynamik, G. M. Conwell, 249.

Neugebauer, O. Die Grundlagen der Aegyptischen Bruchrechnung, F. CAJORI, 371.

d'Ocagne, M. Esquisse d'Ensemble de la Nomographie, L. I. Hewes, 629.

Osgood, W. F. Advanced Calculus, D. R. Curtiss, 241.

- Lehrbuch der Funktionentheorie, zweiter Band, erste Lieferung, E. B. VAN VleCK, 358.

Pasch, M. Vorlesungen über neuere Geometrie (zweite Auflage), mit einem Anhang von M. Dehn, T. R. Hollcroft, 785.

Peirce, B. O. Mathematical and Physical Papers, 1903-13, E. P. Adams, 496.

Plessner, A. See Schlesinger, L.

Rabaté, G. See Bouligand, G.

Reymond, A. History of the Sciences in Greco-Roman Antiquity, translated by R. Gheury de Bray, with preface by L. Brunschvicg, D. E. Sмiтh, 783.

Riemann (B.) und Weber (H.). Die Differential- und Integralgleichungen der Mechanik und Physik (siebente Auflage von Partiellen Differentialgleichungen der Mathematischen Physik), herausgegeben von P. Frank und R. von Mises, erster (mathematischer) Teil, H. J. EтTLINGER, 365.

Rothe, R. Höhere Mathematik (zweite Auflage), Teil I, T. H. HildeBRANDT, 791.

Schlesinger, L., und Plessner, A. Lebesguesche Integrale und Fouriersche Reihen, H. J. Ettlinger, 110.

Schoenflies, A. Einführung in die Analytische Geometrie, J. I. Tracey, 627.

Sommerfeld, A. Three Lectures on Atomic Physics, translated by H. L. Brose, R. D. Carmichael, 794.

Spielrein, J. Vektorrechnung (zweite Auflage), F. D. Murnaghan, 787.

Stenström, O. Synthetische Untersuchungen des Systems von 27 Geraden einer Fläche dritter Ordnung, E. S. Allen, 621.

Sternberg, W. Potentialtheorie, C. A. Sноок, 370. 
Tietze, H. See Hahn, H.

Valiron, G. Théorie Générale des Séries de Dirichlet, L. L. Smail, 630.

Walmsley, C. An Introductory Course of Mathematical Analysis, H. BAteMAN, 498.

Weber, H. See Riemann, B.

Whetham, M. D. See Whetham, W. C. D.

Whetham, W. C. D., and Whetham, M. D., editors. Cambridge Readings in the Literature of Science, U. G. Mitchell, 792.

Willers, F. A. Mathematische Instrumente, A. Емсн, 371.

Zaremba, S. La Logique des Mathématiques, B. A. Bernstein, 629.

Zoretti, L. Leçons de Mathématiques Générales (deuxième édition), W. R. Longley, 781.

Errata, 268, 492, 832.

Index of Volume XXXIII, 820.

New Publications, 125, 254, 380, 509, 635, 800.

Notes, 123, 252, 373, 499, 631, 795.

Papers Read Before the Society and Subsequently Published, Thirty-Sixth Annual List of, 805.

\section{Notes AND OTHER ITEMS}

Academies, Associations, Congresses, and Societies:

American Mathematical Society: Annual Meeting, 373; Auditors' Report, 131; Bequests, 401; Budget, 131; Bulletin, 123, 401; By-Laws, 664; Colloquia, 6, 133,401,499; Committee on Cole Prize, 133; on Newton Celebration, 132; on Nominations, 401; Election of Officers, 132; Endowment Fund, 131; Gibbs Lecture, 6, 133, 401, 501, 644; Librarian's Report, 131; New Members Admitted, 5, 130, 131, 400, 642; Office, 373; Participation in American Journal of Mathematics, 1, 644; Representatives on Council of American Association for the Advancement of Science, 133; on Editorial Board of Annals of Mathematics, 133, 500; on International Mathematical Union, 644; on National Research Council, 133; San Francisco Section, Officers, 2; Statistics, 131; Summer Meeting, 133, 401; Transactions, 123, 373, 499; Treasurer's Report, 131; Visiting Lectureship, 644.

Association for the Advancement of Science, American, 123, 124, 133, 134, 373; British, 795.

American Association of University Women, 501; American Society of Civil Engineers, 374; American Society of Mechanical Engineers, 373; Astronomical Society of the Pacific, 374; Belgian Academy, 631; Bologna Academy, 500; British Institution of Electrical Engineers, 374; Deutsche Mathematiker-Vereinigung, 631; Deutsche Physikalische Gesellschaft, 631; Franklin Institute, 631; Gesellschaft für Angewandte Mathematik und Mechanik, 631; Gesellschaft für Technische Physik, 631; History of Science Society, 500; International Geodetic and Geophysical Union, 631; International Mathematical Congress, 252,373; Italian Society of Sciences, 374; Leningrad Physico- 
Mathematical Society, 252; London Mathematical Society, 252; Mathematical Association of America, 500; Mathematical Association (British) 123, National Research Council, 133; New York Academy of Sciences, 253; Paris Academy of Sciences, 252; Reale Accademia dei Lincei, 631; Royal Academy of Sciences of Holland, 500; Royal Aeronautical Society, 631; Royal Astronomical Society, 374; Royal Society of Edinburgh, 795.

Books, Announcement of New, 252, 499.

Doctorates in Mathematics, American, 374, 502.

Fellowships: Brackett, 501; Guggenheim, 501; Sharpe, 633.

Journals:

American Journal of Mathematics 1, 123, 499, 644, 795; American Mathematical Monthly, 124; Annals of Mathematics, 133, 252, 499, 631, 795; Bulletin of the American Mathematical Society, 123, 401; Crelle's Journal, 123; Leningrad Physico-Mathematical Society Journal, 252; Transactions of the American Mathematical Society, 123, 373, 499.

Papers and Communications Presented to the Society, Authors:

Adams, C. R., 135, 136; Alexander, J. W., 412; Anderson, N. L., 163; Atanasoff, J. V., 164; Ayres, W. L., 8, 263, 266, 266, 410, 410, 657, 661.

Babb, M. J., 412; Barnett, I. A., 398; Barter, J. D., 269, 269, 269; Beal, F. W., 140; Bell, E. T., 3, 3, 269, 513, 513, 514, 664; Bernstein, B. A., 3; Besicovitch, A. S., 652; Biggerstaff, J., 521; Birkhoff, G. D., 10, 134, 144, 659, 660; Blichfeldt, H. F., 409; Blumberg, H., 146, 146, 147, 392, 392; Brahana, H. R., 386; Brand, L., 398; Bray, H. E., 395, 411; Brown, E. P., 136; Buchanan, D., 514; Buchanan, H. E., 653, 654.

Cairns, W. D., 270; Cajori, F., 3, 3, 3, 270, 270, 270; Camp, B. H., 151; Camp, C. C., 145, 397, 652; Campbell, A. D., 157; Campbell, J.W., 521; Carpenter, A. F., 514; Chittenden, E. W., 385; Coble, A. B., 157, 389; Colpitts, J. T., 164; Conkwright, N. B., 394; Copeland, A. H., 396; Court, N. A., 648; Cramlet, C. M., 264.

Davis, D. R., 514, 514, 515; Davis, H. T., 158, 391; Deutsch, H., 515; Dickson, L. E., 160, 161, 161, 389, 389, 390, 390, 650, 650, 650, 650, 662; Dines, L. L., 515, 515, 646, 646; Dodd, E. L., 159, 407; Doggett, W. B., 516; Dorroh, J. L., 656; Douglas, J., 8, 8, 143, 259, 259, 404, 404, 404, 405; Drach, L., 12, 412.

Edington, W. E., 157; Elliott, W. W., 142; Emch, A., 656; Ettlinger, H. J., 9, 164, 258; Evans, G. C., 145, 162, 396, 660, 660.

Flanders, D. A., 411; Foraker, F. A., 139; Ford, W. B., 646; Forsyth, C. H., 135; Fort, T., 146; Foster, M. C., 139; Franklin, P., 10, 10, 149, 151, 267, 404.

Gaba, M. D., 163; Gage, W. H., 516; Garabedian, C. A., 135; Garver, R., 264, 264, 408, 408, 408; Gehman, H. M., 7, 147; Gergen, J., 396, 655; Gillespie, D. C., 654; Glenn, O. E., 149, 260, 390; Gourin, E., 152, 259; Graustein, W. C., 140; Graves, L. M., 9, 654; Griffin, F. L., 516; Gronwall, T. H., 12, 12, 258, 258, 258; Grove, V. G., 156, 649. 
Haack, B. G., 516; Hancock, H., 149, 149; Haskell, R. N., 407; Hazlett, O. C., 148, 148, 148; Hedrick, E. R., 3, 270; Heineman, E. R., 651; Hildebrandt, T. H., 646, 647; Hofmann, L., 399; Holl, D. L., 162; Hollcroft, T. R., 10, 138, 264, 403, 647; Hosford, H. M., 142; Hotelling, H., 4, 271, 648, 652, 653; Huber, C. M., 11.

Ingold, L., 164.

Jackson, D., 140, 398, 646; James, G., 271; Jerbert, A. R., 516.

Kasner, E., 267, 267, 409; Keller, E. G., 390; Kempner, A. J., 650, 653; Kimball, B. F., 7; Kline, J. R., 401; Koopman, B. O., 6, 137, 405, 406; Kormes, M., 146.

LaMer, V. K., 12; Langer, R. E., 136, 136, 136, 646; Langford, C. H., 151, 405, 658; Latimer, C. G., 649, 649; Lefschetz, S., 11, 386, 391; Lehmer, D. H., 271, 272; Lehmer, D. N., 4; Levy, H., 138; Logsdon, M. I., 387, 387; Lotka, A. J., 403.

McDonald, J. H., 2; McVey, P., 412; MacDuffee, C. C., 389; MacNeish, H. F., 152; Mandelbrojt, S., 145; Manning, W. A., 4, 160, 409; March, H. W., 157; Maria, A. J., 412, 412; Mathews, R. M., 387; Mears, F. M., 659; Merriman, G. M., 407, 658; Miller, G. A., 160, 649; Moore, L. T., 402, 403; Moore, R. L., 8, 141, 521, 656; Moore, T. W., 260; Moritz, R. E., 517, 517, 517; Morley, F., 389; Morse, M., 150; Moulton, E. J., 161; Mullings, M. E., 395; Murnaghan, F. D., 134, 138, 141, 407; Musselman, J. R., 135, 408.

Neelley, J. H., 139, 402; Neikirk, L. I., 272, 517; Nowlan, F. S., 517, 518, 518; Nyswander, J. A., 156.

Parkinson, G. A., 647; Pennock, D., 518; Pettit, H. P., 386, 386; Pfeiffer, G. A., 410; Pierpont, J., 11, 148, 152, 261, 405; Putnam, R. G., 146.

Rainich, G. Y., 155, 157, 391, 655; Raudenbush, H. W., 260; Reynolds, C. N., 150; Rice, L. H., 10; Richmond, D. E., 411; Rider, P. R., 391; Ritt, J. F., 6, 7, 152; Roever, W. H., 134; Rollins, M., 518; Roos, C. F., 159, 159, 393, 393, 661, 660; Rosebrugh, T. R., 268; Roth, W. E., 651; Rutt, N. E., 411.

Schelkunoff, S. A., 647; Schmidt, R., 265; Schwatt, I. J., 150; Serghiesco, S., 7, 406; Sharpe, F. R., 649; Shaub, H. C., 10; Shaw, J. B., 398; Sheffer, I. M., 155; Shohat, J. A., 158, 394, 645; Sibert, H. W., 142; Silverman, L. L., 142, 267; Simmons, H. A., 399; Smail, L. L., 141; So, H. S., 518; Stone, M. H., 11, 261, 261; Struik, D. J., 257, 410; Swift, E., 144.

Tamarkin, J. D., 136, 137, 267; Taylor, J. H., 156, 393; Teach, V. B., 8; Thomas, J. M., 265; Trjitzinsky, W. J., 155, 518, 519, 519, 519, 519, 520, 661, 661; Turner, J. S., 163, 399.

Uspensky, J. V., 158, 158, 394, 662, 662.

Vandiver, H. S., 147, 152, 651, 651, 652, 652.

Wahlin, G. E., 163; Wall, H. S., 397; Walsh, J. L., 150, 658, 658; Ward, L. E., 391, 392; Wear, L. E., 4; Weaver, J. H., 648; Webber, W. P., 659; Weisner, L., 9, 260, 402, 402, 402; Wells, V. H., 139; Wheeler, A. P., 664; Whyburn, G. T., 153, 154, 154, 154, 262, 262, $262,263,388,389,408,408,520,520,522,656,657,657$; Whyburn, 
W. M., 9, 155, 263, 263, 394, 395, 659; Widder, D. V., 143, 143, 143, 265, 396, 655, 655; Wiener, N., 261, 261, 261, 265, 266, 410; Wilder, R. L., 147, 263, 388, 388; Williams, A. R., 272; Williams, H. B., 133; Williamson, J., 161; Wilson, W. A., 8, 658; Winger, R. M., 521; Wong, B. C., 272.

Young, J. W., 265.

Zeldin, S. D., 140.

Personal Notes:

von Abo, C. V., 374; Adams, W. S., 123; Ahrens, W., 634; Alayrac, A., 252; Ames, J. S., 796; Anderson, L. A., 379; Archibald, R. C., 500; Archibald, R. G., 634; Arnold, H. E., 796; Arrhenius, S., 632; Artin, E., 253; Auerbach, F., 634; Aylesworth, E. F., 375.

Babcock, W., 375; Bachelier, 796; Bagby, L. C., 633; Bagnera, G., 634; Bailey, H. W., 375; Baire, R., 253; Baker, Frances, 798; Bamforth, F. R., 798; Barnard, R. W., 375, 797; Bartke, Walter, 796; Barton, M. H., 375; Bell, E. T., 133, 499, 500; Benedict, H. Y., 797; Bennett, A. A., 506; Benton, T. C., 634; Bernstein, S., 252; Berthelot, D., 508; Bescovitch, A.S., 633; Bibb, S. F., 379; Billings, H. C., 798; Birkhoff, G. D., 1, 124, 134, 500, 632; Black, F. L., 375; Black, H. L., 375; Blaschke, E., 124; Blaschke, W., 373; Blichfeldt, H. F., 133, 133; Bloch, E., 374; Blue, A. H., 797; Bohr, N., 796; Borden, R. F., 508; Borel, E., 500; Bortolotti, E., 631; Bose, A. C., 799; Bowie, W., 631; Boyce, M. G., 798; Brackett, F. P., 632; Brauer, R., 501; von Brill, A., 500; Brink, R.W., 123; Brinkmann, H.W., 506; Brodetsky, S., 124; de Broglie, L., 252; Brown, E. W., 401, 500, 501, 644; Browne, E. T., 375; Bruhat, G., 253; Bucherer, A. H., 508; Bullard, W. G., 634; Burmester, L., 634; Burnett, D., 500; Burnside, W., 799; Bussey, W. H., 124; Butler, L. G., 253.

Cajori, F., 500; Camp, C. C., 797 ; Campbell, A. D., 253; Campbell, D. F., 797; Campbell, W. B., 797; Carathéodory, C., 501; Cathcart, G. L., 634; Cecioni, F., 374; Chapman, S., 252; Chisini, O., 374; Coble, A. B., 1, 6, 133, 379; Cohen, A., 1; Comessatti, A., 374; Compton, A. H., 123; Compton, K. T., 124; Conkwright, N. B., 375; Coolidge, J. L., 506; Cooper, A. E., 375; Copeland, A. H., 375; Cousin, P., 374; Cramlet, C. M., 375; Crandall, I. B., 799; Crelle, A. L., 123; Crum, W. L., 797; Cruse, S. R., 379; Curie, 632.

Dantzig, T., 506; Darkow, M., 798; Davis, D. R., 253; Davis, H. T., 375; Debye, P., 253; Dederick, L. S., 633; Dehn, E., 253; Demos, M. S., 375, 508; Dickson, L. E., 123; Dixon, A. L., 252; Doner, R. D., 375; Dresden, A., 133, 379, 644; Dwyer, P. S., 253.

Eberhard, V., 634; Eckert, C. H., 501; Eddington, A. S., 632; Eells, W. C., 506; Einstein, A., 632, 796, 796; Elliott, W. W., 253, 633; Emch, Arnold, 797; Engstrom, H. T., 508; Ettlinger, H. J., 797; Evans, G. C., 1, 133, 133, Everett, H. S., 797.

Fabry, C., 796; Farnum, F., 375; Feigl, G., 501; Feld, J., 634; Fermi, E., 374; Fields, J. C., 252; de Fontviolant, E. B., 252; Ford, W. B., 500; Fort, T., 133, 506; Foster, M. C., 797; Fraleigh, P. A., 
633; Franck, J., 124; Franklin, P., 501; Fréchet, M., 253; Freeman, G. H., 798; Frink, O., 375.

Gaba, M. G., 124; Garabedian, H. L., 634; Garretson, W. V. N., 797; Garver, R., 375, 798; Gavett, G. I., 633; Gehman, H. M., 506; Georges, J. S., 375; Gibbs, J. W., 502; Gibson, G. E., 501; Gillespie, D. C., 133, 500; Gmeiner, A., 634; Gockel, A., 798; Goldstein, S., 500; Goodier, J. N., 795; Goodwin, H. B., 508; de la Goupillière, H., 379; Graesser, R. F., 375; Gray, M. C., 375; Green, R. L., 506; Greenhill, G., 379; Griffiths, L. W., 798; Grossman, L., 634; Guggenheim, D., 253, 378, 501; Guye, C. E., 374.

Hadamard, J., 500; Hadlock, E. H., 798; Hagen, J. G., 632; Hale, G. E., 631; Hardy, G. H., 252, 632, 796; Hargreaves, J., 795; Hart, W. L., 124, 133, 500; Hertz, G., 124; Hickson, A. O., 508; Hilbert, D., 500; Hill, G. A., 799; Hill, L. S., 375; Hilton, H., 252; Hodge, W. V. D., 500; Hölder, O., 796; Hosford, H. M., 375; Houston, W. V., 501; Howarth, H. A. S., 373; Hoyt, F. C., 501; Huber, C. M., 376, 506; Hughes, J C., 797; Hulbert. L. S., 506; Hutchinson, J. I., 632.

Ingraham, M. H., 507, 645.

Jackson, D., 123; Jackson, R. L., 798; Jeffery, R. L., 798; Joffe, S. A., 131; Johnson, M., 798; Johnson, W. W., 634; Johnston, F. E., 376: Jordan, P., 501; Julia, G., 252; Justice, H., 633.

Kamke, E., 253; Kellogg: O. D., 507; Kenny, M. V., 798; Keyser, C. J., 507; Kimball, A. L., 373; Kimball, B. F., 376; Kindle, J. H., 633; Kline, J. R., 499; Koebe, P., 796; Kokomoor, F. W., 376; Koopman, B. O., 376, 634; Krull, W., 632.

Ladd-Franklin, C., 502; Lafay, A., 633; Lamb, H., 123; Lambert, W. D., 631; Lanczos, K., 501; Landau, E., 500; Langer, R. E., 133, 500, 507; Langford, C. H. 797; Larmor, J., 795; Lennard-Jones, J. E., 501; Lense, J., 632; Lenzen, V.F., 501; Lettenmeyer, F., 796; Levy, H., 507; Linehan, P. H., 633; Littlewood, J. E., 252; Logsdon, M. I., 124; Loud, F. H., 508.

McFarlan, L. H., 797; MacCreadie W. T., 797; MacRobert, T. M., 796; Mader, O., 501; Maizlish, I., 507; Mammana, G., 501; Mason M., 632; Maurer, L., 634; Meacham, E. D., 507; Mears, F. M., 797; de Mendizàbal Tamborrel, J.. 253; Menzel, D. H., 253; Meredith, C. A., 500; Merriman, G. M., 502; Mertens, F., 508; Messick, C. A., 798; Metz, M L., 633; Michal, A. D., 507; Michelson, A. A., 632, 632; Miller, D. C., 632; Miller, E. B., 797; Millikan, R. A., 500, 796; Milne, T. H., 633; Mitchell, H. H., 133; Mittag-Leffler, G., 632, 798; Montel, P., 252; Moody, E. I., 798; Moore, E. H., 632; Moore, R. L., 133, 401; Moore, T. W., 253; Morenus, E. M., 501; Morley, F., 1; Moulton, F. R., 796; Murnaghan, F. D., 499.

Nansen, F., 796; Nassau, J. J., 507; Neelley, J. H., 633; Nelson, C. A., 633; Nernst, W., 632; Newman, H. A., 796; Newton, I., 123, 500; Nowlan, F. S., 124; Noyes, A. A., 123.

Olds, G. D., 632; Ore, O., 507.

Palmer, C. I., 797; Pars, L. A., 796; Patterson, B. C., 376; Perrin, J., 124; Persico, E., 374; Phalen, H. R., 376; Pierpont. J., 373; 
Pincherle, S., 373; Pixley, H., 798; Planck, M., 374, 500, 631, 633; Popoff, K., 252; Pounder, I. R., 376; Prandtl, L., 631; Pryde, H. H., 376; Pupin, M. I., 632.

Quemper de Lanascol, A., 252.

Raimondi, E., 501; Ranum, A., 632; Raudenbush, H. W., 634; Rawles, T. H., 508; Raynor, G. E., 507; Reddick, H. W., 131; Reeve, W. D., 507; Reinsch, B. P., 634; Richmond, D. E., 376; 507; Riddle, G., 253; Ritt, J. F., 507; Roos, C. F., 376; Runge, C., 379; Russell, H. N., 501.

Sansone, G., 374; Schaub, H. C., 798; Scheffers, G., 796; Schlesinger, F., 252, 374; Schrödinger, E., 253, 797; Schwartz, P. D., 376; Schur, F., 796; Serini, R., 374; Sharpe, H. D., 633; Shook, C. A., 507; Silberfarb, Samuel, 798; Siff, L., 124; Slepian, J., 379; Slotnick, M. M., 376; Smith, D. E., 500; Smith, E. S., 633; Smith H. L., 376; Smith, P. A., 376, 634; Sommerfeld, A., 633; Stark, M. E., 376, 798; Stehn, J., 508; Stevenson, G., 376; Stone, M. H., 376, 508; Study, E., 796; Swingle, P. W., 798.

Tam,V.A., 798; Tamarkin, J. D., 123, 507; Terami, T., 376; Thieme, F., 634; Thomas, J. M., 798; Thompson, H. D., 799; Thomson, E., 374; Thomson, J. J., 123; Thurston, H. S., 507; Timoshenko, S., 373; Tonelli, L., 631; Torrance, C. C., 798; Trjitzinsky, W. J., 798; Tschuprow, A., 124; Tudor, J. H., 124; Turner, H. H., 374.

Upton, C. B., 379.

Vallarta, M. S., 501; Vandiver, H. S., 501; Veblen, O., 123; Vedova, G. C., 634; Villat, H., 633; Virata, E. T., 376.

van der Waerden, B. L., 796; Wagner, P. S., 376; Walter, A., 634; Warren, L. A. H., 798; Wassmuth, A., 799; Watson, G. N., 252; Wedderburn, J. H. M., 123, 133, 401; Weisner, L., 798; Western, A. E., 252; Wheeler, A. P., 500; White, F. P., 252; Whitehead, A. N., 632; Whitford, E. E., 634; Whittaker, E. T., 795; Wien, W., 374; Wiksell, S. D., 379; Wilkins, P. D., 798; Williams, B., 634; Williams, F. G., 798; Williams, H. B., 6, 133; Wilson, C. R., 798; Wilson, E. B., 631; Wilson, W. A., 507; Wilson, W. H., 798; Worth, C. R., 798.

Young, C. A., 501.

Zinszer, H. A., 634; Ziwet, A., 632.

Prizes and Medals:

Abbe, 374; Ackermann-Teubner, 373; American Association for the Advancement of Science, 124, 134; American Society of Civil Engineers, Collingwood Prize, 374; Astronomical Society of the Pacific, Bruce Medal, 374; Belgian Academy, 631; Bologna Academy, Merlani Prize, 500; British Institution of Electrical Engineers, Faraday Medal, 374; Cambridge University, 500, 795; Cole, 133;Franklin Institute, 631; Italian Society of Sciences, 374; Lorentz, 500; New York Academy of Sciences, Morrison Prize, 253; Nobel, 124; Paris Academy, 252; Reale Accademia dei Lincei, 631; Royal Aeronautical Society, 631; Royal Astronomical Society, 374; Royal Society of Edinburgh, Scott Prize, 795. 
Universities and Technical Schools:

Brown, 376, 502, 633;

Michigan, 253, 377, 504;

California, 502;

Minnesota, 378;

Cambridge, 500, 795;

Ohio State, 378, 505;

Chicago, 376, 502;

Pennsylvania, 378;

Cincinnati, 503;

Pittsburgh, 379;

Columbia, 377, 503;

Princeton, 505;

Cornell, 503, 632;

Harvard, 503;

Illinois, 377, 504;

Rice, 505;

Stanford, 378;

Iowa, 377, 504;

Texas, 378, 506;

Wisconsin, 378;

Johns Hopkins, 1, 132, 377, 504;

Yale, 502, 506.

\section{ERRATUM}

On page 631 change Edmund $B$. Wilson, in line 23, to Edwin B. Wilson. 